\title{
Identify COVID-19 from chest X-ray images by Artificial Intelligence
}

\author{
Abdelrahman Abdelnasser Gamal Mohamed \\ "STEM High School for Boys \\ $+201554891828$ \\ 18036@stemegypt.edu.e
}

DOI: 10.29322/IJSRP.10.08.2020.p10462

http://dx.doi.org/10.29322/IJSRP.10.08.2020.p10462

\begin{abstract}
Artificial intelligence is a breakthrough in technology. Artificial intelligence provided many services in various fields, especially in the field of medicine, where he anticipated the epidemic and presented many solutions to our help in overcoming it. I was able to develop a project to obtain the highest accuracy in identifying people affected by Covid-19.

Seeking the best and cheapest way to diagnose individuals with COVID-19 infections or suspects. Or finding new viral and bacterial pneumonia (MERS, SARS, and ARDS). In Egypt (Developing Countries), the polymerize chain reaction (PCR) test takes around 48 hours to get the result, and the result isn't always accurate and costs a lot of money in the range of $1000-2000$, so many people can't afford it. Also, these tests aren't available in many governments so people could die for not having these tests, Also, due to the huge amount of infected people, there's a lack of medicine in the local pharmacies.

Due to the lack of medicine, wrong clinical diagnosis by some doctors, and the enormous amount of money that the country spends on tests and medications, we decided to create a web application that uses Deep Learning and Artificial intelligence algorithms to analyze " CT chest - Chest X-ray " for suspected individuals and getting the results immediately and efficiently.
\end{abstract}

Index Terms- Artificial Intelligence, deep neural network Machine Learning, COVID-19, CT chest, Chest X-ray, Deep Learning, Data Science, Algorithms, Egypt, Polymerise chain reaction (PCR), Developing Countries.

\section{INTRODUCTION}

Artificial intelligence systems had previously observed the unknown form of pneumonia in the People's Republic of China before the world had even become aware of the threat posed by the coronavirus (COVID-19). Since the epidemic has now become a global pandemic, AI tools and technology will help policymaking, the medical community, and society as a whole navigate each stage of the crisis and its consequences: identification, preventing, responding, recovering, and accelerating science.

AI technologies and methods can help policymakers and the healthcare community understand COVID-19 viruses and expedite therapy research through rapid analyzes of detailed research data. Text mining instruments for AIs and data can reveal the history of the virus, transmission and diagnosis, management measures, and the lessons of earlier epidemics.

\section{Problem}

Seeking the quickest and cheapest route for an investigator, COVID-19-infected person (MERS, SARS, and ARDS) or suspected COVID-19-pulmonary person where Egypt did not take modern technology to monitor patients because there is minimal technology across the globe to achieve the precise result, so Egypt turned to an alternative solution like polymerize chain reaction (PCR), The analysis is carried out by taking a blood sample from the suspect who is infected with the virus, and the result will appear within two days of the procedure and it is expensive and, limited in Governments after we conformed from an online social survey where we found the minimum to releases the results are two days and the cost in the range from 1000 to 2000 , and it isn't accurate where it Shows positive results in 30\% of cases Coronavirus, and this percentage it is lower than anther original tests.

There are three kinds for testing are, the first Polymerise Chain Reaction (PCR), it is available in many countries but it very Expensive, and take a long time to see the result, and accurate by $30 \%$, the second type is CDC tests are provided to U.S. state only and local public health laboratories and Department of Defense laboratories that were either previously qualified for being able to perform a similar type of test used to detect influenza or have been recently approved by their state public health laboratory for SARS-CoV-2 and COVID disease testing. the third is FDA tests, there are a group of tests like Diagnostic test, viral test, molecular test, nucleic acid amplification test (NAAT), RT-PCR test, LAMP test, and it didn't available in a lot of locations and the period to get the result from one day (some locations) or up to a week, and it very expensive, so we start to find another solution solve disadvantages of the three tests, to cheap and available in all Countries, and get the result in the current time, get the result in the current time, and there are many types of testing but it is common.

trying to find another way More Accurate, Cheaper than PCR by building an AI website application use a public open dataset of chest X-ray and CT images of patients which are positive or suspected of COVID-19 or other viral and bacterial pneumonia (MERS, SARS, and ARDS.) as shown in figure (1).

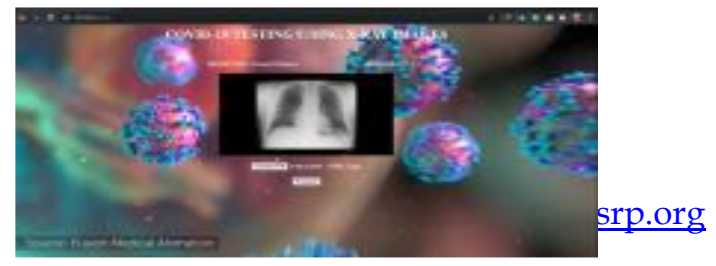


With the Chest X-Ray dataset, develop a Machine Learning Model to classify the X Rays of Healthy vs Pneumonia (Corona) affected patients \& this model powers the AI application to test the Corona Virus in Faster Phase Data will be collected from public sources as well as through indirect collection from hospitals and physicians, where Corona COVID19 virus affects the respiratory system of healthy individual \& Chest X-Ray which is shown in figure (2) is one of the important imaging methods to identify the coronavirus.

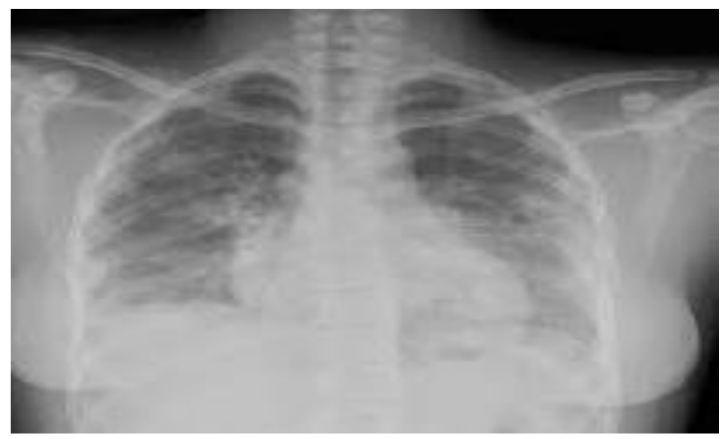

\section{Background}

In the context of a COVID-19 pandemic, we want to improve prognostic predictions to triage and manage patient care. Data is the first step to developing any diagnostic/prognostic tool. While there exist large public datasets of more typical chest X-rays from the NIH [Wang 2017], Spain [Bustos 2019], Stanford [Irvin 2019], MIT [Johnson 2019] and Indiana University [Demner-Fushman 2016], there is no collection of COVID-19 chest X-rays or CT scans designed to be used for computational analysis.

The 2019 novel coronavirus (COVID-19) presents several unique features Fang, 2020, and Ai 2020. While the diagnosis is confirmed using polymerase chain reaction (PCR), infected patients with pneumonia may present on chest X-ray and computed tomography (CT) images with a pattern that is only moderately characteristic for the human eye $\mathrm{Ng}, 2020$. In late January, a Chinese team published a paper detailing the clinical and paraclinical features of COVID-19. They reported that patients present abnormalities in chest CT images with most having bilateral involvement Huang 2020. Bilateral multiple lobular and subsegmental areas of consolidation constitute the typical findings in chest CT images of the intensive care unit (ICU) patients on admission Huang 2020. In comparison, nonICU patients show bilateral ground-glass opacity and subsegmental areas of consolidation in their chest CT images Huang 2020. In these patients, later chest CT images display bilateral ground-glass opacity with resolved consolidation Huang 2020.

This information from Ieee8023/covid-chestxray-dataset.

\section{Idea}

First, identify the problem which conformed by the online survey and try to solve it by creating new idea solve the disadvantage of the main problem as we explained before, I develop an AI web application by that using Deep Learning and Artificial intelligence to analyze " CT chest - Chest X-ray " by using datasets which X-ray images obtained from two different sources were used for the diagnosis of COVID-19. A COVID-19 X-ray image database was developed by Cohen JP using images from various open access sources.

This database is constantly updated with images shared by researchers from different regions, to can define if the input is Xray for chest or not by learning the stats of PA, AP, and AP Supine views, and use Convolutional type Neural Network to identify Covid-19 positive patients using X-Ray images, then deploy the previously CNN model and Training the CNN, and create Convolutional Neural Network Architecture, The model is sensitive in detecting pneumonia disease. Although the model can predict pneumonia positively and marked as no findings in the dataset.

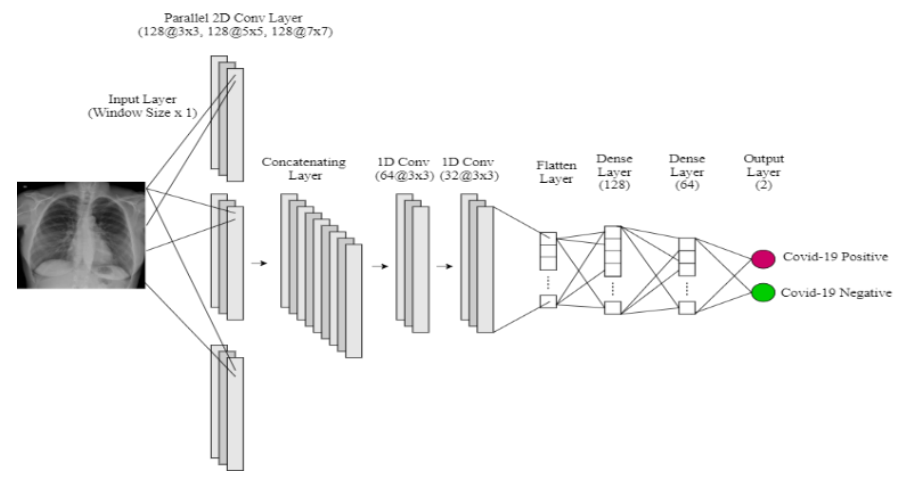

In a web application using a Python backend with a Flask web development framework and other Modules like TensorFlow, Keras, and OpenCV modules to connect ipynb with python then Create the data set and connected it by the database for Positive X-Ray images the data Database collected from Joseph Paul Cohen. Postdoctoral Fellow, Mila, the University of Montreal for the dataset below for corona dataset \& $80 \%$ dataset collected from different sources like IEEE COVID-chestx-raydataset and Kaggle Corona Hack -Chest X-Ray-Dataset, then after finished the backend connected it with The frontend of the website is created with HTML and JavaScript and use some library like Bootstrap, and jQuery.

\section{Conclusion}

In this study, we have proposed a deep learning-based model to detect and classify COVID-19 cases from X-ray images. Our model is fully automated with an end-to-end structure without the need for manual feature extraction. Our developed system can perform binary and multi-class tasks with accuracy in the range of $98.08 \%$ to $87.02 \%$, respectively. Expert radiologists assess the efficiency of the built model and can 
compare it with a larger database. This program can be used to resolve the scarcity of radiologists at remote sites in countries affected by COVID-19. These models may also be used to treat other chest diseases such as tuberculosis and pneumonia. The use of a small number of COVID-19 X-ray images is a drawback of the study. With more photos from our local hospitals, we plan to make our model more reliable and accurate.

\section{REFERENCES}

I. Ai, T., Yang, Z., Hou, H., Zhan, C., Chen, C., Lv, W., . . . Xia, L. (2020). Correlation of Chest CT and RT-PCR Testing in Coronavirus Disease 2019 (COVID-19) in China: A Report of 1014 Cases. Radiology, 200642. DOI:10.1148/radiol.2020200642

II. Abdelrahman, A. A. (2020). Artificial Intelligence and Humans. International Journal of Scientific and Research Publications (IJSRP), 10(3), 548-551. DOI:10.29322/ijsrp.10.03. 2020.p9970

III. Ieee8023. (2020). Ieee8023/covid-chestxray-dataset. Retrieved July 19, 2020, from https://github.com/ieee8023/covid-chestxray-dataset

IV. Selvan, R., Dam, E., Detlefsen, N., Rischel, S., Sheng, K., Nielsen, M., \&amp; Pai, A. (2020, July 07). Lung Segmentation from Chest X-rays using Variational Data Imputation. Retrieved July 19, 2020, from https://arxiv.org/abs/2005.10052

V. Signoroni, A., Savardi, M., Benini, S., Adami, N., Leonardi, R., Gibellini, P., . . . Farina, D.(2020, June 08). End-to-end learning for a semiquantitative rating of COVID-19 severity on Chest X-rays. Retrieved July 19, 2020, from https://arxiv.org/abs/2006.04603

VI. Global research on coronavirus disease (COVID-19). (2020). Retrieved July 19, 2020, from https://www.who.int/emergencies/diseases/novelcoronavirus-2019/global-research-on-novel-coronavirus2019-ncov

VII. Cohen, J., Dao, L., Morrison, P., Roth, K., Bengio, Y., Shen, B., . . . Duong, T. (2020, June 30). Predicting COVID-19 Pneumonia Severity on Chest X-ray with Deep Learning. Retrieved July 19, 2020, from https://arxiv.org/abs/2005.11856

VIII. Ieee8023. (n.d.). Ieee8023/covid-chestxray-dataset. Retrieved July 19, 2020, from https://github.com/ieee8023/covid-chestxray-dataset

IX. Tartaglione, E., Barbano, C., Berzovini, C., Calandri, M., \&amp; Grangetto, M. (2020, April 11). Unveiling COVID19 from Chest X-ray with deep learning: A hurdles race with small data. Retrieved July 19, 2020, from https://arxiv.org/abs/2004.05405

X. Maguolo, G., \&amp; Nanni, L. (2020, May 19). A Critic Evaluation of Methods for COVID-19 Automatic Detection from X-Ray Images. Retrieved July 19, 2020, fromhttps://arxiv.org/abs/2004.12823

XI. Commissioner, O. (2020). Coronavirus Testing Basics. Retrieved July 20, 2020, from https://www.fda.gov/consumers/consumerupdates/coronavirus-testing-basics
XII. CDC Diagnostic Tests for COVID-19. (2020, July 03). Retrieved July 20, 2020, from https://www.cdc.gov/coronavirus/2019ncov/lab/testing.html

XIII. The first initiative of publicly deploy whole CT scans of COVID-19. (n.d.). Retrieved July 20, 2020, from https://coronacases.org/

XIV. Ozturk, T., Talo, M., Yildirim, E., Baloglu, U., Yildirim, O., \& Rajendra Acharya, U. (2020, June). Automated detection of COVID-19 cases using deep neural networks with X-ray images. Retrieved July 28, 2020, from https://www.ncbi.nlm.nih.gov/pmc/articles/PMC7187882/

XV. Abdelrahman, A. A. (2020). Identify COVID-19 from chest X-ray images by Artificial Intelligence. Retrieved July 28, 2020, from https://github.com/kabaka121212/IdentifyCOVID-19-from-chest-X-ray-images-by-ArtificialIntelligence 